\title{
X-ray diffraction studies on crystallite size evolution of CoFe2O4 nanoparticles prepared using mechanical alloying and sintering.
}

\begin{abstract}
Nanosized cobalt ferrite spinel particles have been prepared by using mechanically alloyed nanoparticles. The effects of various preparation parameters on the crystallite size of cobalt ferrite which includes milling time; ball-to powder weight ratio (BPR) and sintering temperature, were studied using X-ray diffractometer (XRD). Scherrer's equation was used to study the crystallite size evolution of the as-prepared materials. The results of the as-milled sample revealed that both milling time and BPR plays a role in determining the crystallite size of the milled powder. However, where sintering is involved, the sintering temperature results in grain growth, and thus plays a dominant role in determining the final crystallite size of the samples sintered at higher temperature (above $900{ }^{\circ} \mathrm{C}$ ). From the vibrating-sample magnetometer (VSM) measurement it was observed that the coercivity of the as-milled samples without sintering is almost negligible, which is a type characteristic of superparamagnetic material. However, for the sintered samples, the saturation increases while coercivity decreases with increases sintering temperature.
\end{abstract}

Keyword: Crystallite size; Sintering temperature; X-ray diffraction; Nanoparticles. 